\title{
Discrete-Continuous Symmetrized Sobolev Inner Products
}

\author{
MARÍA ISABEL BUENO ${ }^{1}$, KIL H. KWON ${ }^{2}$ and \\ FRANCISCO MARCELLÁN ${ }^{1}$ \\ ${ }^{1}$ Departamento de Matemáticas, Universidad Carlos III de Madrid, \\ Avenida Universidad, 30. 28911 Leganés, Spain. \\ e-mail: mbueno@math.uc3m.es, pacomarc@ing.uc3m.es \\ ${ }^{2}$ Division of Applied Mathematics, KAIST, 373-1 Kusong-dong Yusong-ku, Taejon(305-701), \\ Korea.e-mail:khkwon@amath.kaist.ac.kr \\ प
}

Abstract. This paper deals with the bilinear symmetrization problem associated with Sobolev inner products. Let $\left\{Q_{n}\right\}_{n=0}^{\infty}$ be the sequence of monic polynomials orthogonal with respect to a Sobolev inner product of order 1 when one of the measures is discrete and the other one is a nondiscrete positive Borel measure. Furthermore, assume that the supports of such measures are symmetric with respect to the origin so that the corresponding odd moments vanish. We consider the orthogonality properties of the sequences of monic polynomials $\left\{P_{n}\right\}_{n=0}^{\infty}$ and $\left\{R_{n}\right\}_{n=0}^{\infty}$ such that $Q_{2 n}(x)=P_{n}\left(x^{2}\right), Q_{2 n+1}(x)=x R_{n}\left(x^{2}\right)$. Moreover, recurrence relations for $\left\{P_{n}\right\}_{n=0}^{\infty}$ and $\left\{R_{n}\right\}_{n=0}^{\infty}$ are obtained as well as explicit algebraic relations between them.

Mathematics Subject Classification (2000): $42 \mathrm{C} 05$.

Key words: Sobolev inner product, orthogonal polynomials, semiclassical linear functional, recurrence relation, symmetrization process.

\section{Introduction}

Let $\mathbf{U}$ be a quasi-definite linear functional defined in the linear space $\mathbb{P}$ of polynomials with real coefficients. Then, there exists a Borel measure $\mu$ supported on the real line such that

$$
\mathbf{U}(p)=\int_{\mathbb{R}} p \mathrm{~d} \mu .
$$

The linear functional $\mathbf{U}$ is said to be symmetric if $\mathbf{U}\left(x^{2 n+1}\right)=0$ for $n \geqslant 0$. In particular, if $\mathbf{U}$ is positive definite and symmetric, the support of the measure $\mu$ is a symmetric set with respect to the origin on the real line. Let $\left\{Q_{n}\right\}$ be the sequence of monic polynomials orthogonal with respect to $\mathbf{U}$. If $\mathbf{U}$ is a symmetric linear functional, then there exists a sequence of monic polynomials $\left\{P_{n}\right\}$ such that

$$
Q_{2 n}(x)=P_{n}\left(x^{2}\right), \quad Q_{2 n+1}(x)=x P_{n}^{*}\left(x^{2}\right),
$$


where $\left\{P_{n}^{*}\right\}$ denotes the sequence of monic kernel polynomials of parameter 0 associated with the sequence $\left\{P_{n}\right\}$. It is well known (Chihara, 1978) that the sequence $\left\{P_{n}\right\}$ is orthogonal with respect to the linear functional $\mathbf{L}$ given by

$$
\mathbf{L}\left(x^{n}\right):=\mathbf{U}\left(x^{2 n}\right), \quad n \geqslant 0 .
$$

Moreover, the sequence $\left\{P_{n}^{*}\right\}$ is orthogonal with respect to the linear functional $\mathbf{L}^{*}$ defined as

$$
\mathbf{L}^{*}(p):=(\mathbf{x L})(p)=\mathbf{L}(x p) .
$$

More explicitly, if the functionals $\mathbf{L}$ and $\mathbf{L}^{*}$ can be expressed, respectively, in terms of the Borel measures $\mathrm{d} \mu_{1}$ and $\mathrm{d} \mu_{2}$ then,

$$
\mathrm{d} \mu_{1}(x)=\mathrm{d} \mu\left(x^{1 / 2}\right), \quad \mathrm{d} \mu_{2}(x)=x \mathrm{~d} \mu_{1}(x) .
$$

If $\mu$ is an absolutely continuous measure, i.e., $\mathrm{d} \mu(x)=\omega(x) \mathrm{d} x$, then $\mu_{1}$ is also an absolutely continuous measure and the corresponding weight function $\omega_{1}$ satisfies

$$
\omega_{1}(x)=x^{-1 / 2} \omega\left(x^{1 / 2}\right) .
$$

It is also well known that the following explicit algebraic relation between the sequences $\left\{P_{n}\right\}$ and $\left\{P_{n}^{*}\right\}$ holds

$$
x P_{n}^{*}(x)=P_{n+1}(x)-\frac{P_{n+1}(0)}{P_{n}(0)} P_{n}(x), \quad n \geqslant 0 .
$$

Furthermore, since the sequences $\left\{P_{n}\right\}$ and $\left\{P_{n}^{*}\right\}$ are orthogonal with respect to linear functionals, both satisfy a three-term recurrence relation.

This linear symmetrization process was extensively studied by Chihara (1978). A similar process can be considered related to bilinear functionals. Let $\mathbf{U}$ be a quasi-definite bilinear functional and let $\left\{Q_{n}\right\}$ be the corresponding sequence of monic orthogonal polynomials. The functional $\mathbf{U}$ is said to be a symmetrized functional if $\mathbf{U}\left(x^{n}, x^{m}\right)=0$ when $n+m$ is an odd number. Notice that now we do not use the term symmetric, since it is already associated with another concept when dealing with bilinear functionals. In such a case, it can be proven that there exist two sequences of polynomials $\left\{P_{n}\right\}$ and $\left\{R_{n}\right\}$ such that

$$
Q_{2 n}(x)=P_{n}\left(x^{2}\right), \quad Q_{2 n+1}(x)=x R_{n}\left(x^{2}\right), \quad n \geqslant 0 .
$$

Then, the following questions arise in a natural way:

1. What are the bilinear functionals such that $\left\{P_{n}\right\}$ and $\left\{R_{n}\right\}$ are the corresponding sequences of monic orthogonal polynomials?

2. Does there exist any explicit algebraic relation between the sequences $\left\{P_{n}\right\}$ and $\left\{R_{n}\right\}$ ?

3. Do the sequences $\left\{P_{n}\right\}$ and $\left\{R_{n}\right\}$ satisfy any kind of recurrence relation with a finite number of terms? 
The answers to the previous questions constitute the so-called bilinear symmetrization process.

In this paper we consider a particular case of symmetrized bilinear functional, the so-called symmetrized Sobolev inner products of order 1 defined as

$$
\langle p, q\rangle_{s}=\int_{\mathbb{R}} p(x) q(x) \mathrm{d} \mu_{0}+\int_{\mathbb{R}} p^{\prime}(x) q^{\prime}(x) \mathrm{d} \mu_{1}, \quad p, q \in \mathbb{P},
$$

where $\mu_{0}$ and $\mu_{1}$ are Borel measures supported on symmetric sets with respect to the origin on the real line so that

$$
\int_{\mathbb{R}} x^{2 n+1} \mathrm{~d} \mu_{k}=0, \quad k=0,1, n \geqslant 0 .
$$

In a previous paper (Bueno and Marcellán, 2003) we studied the symmetrization process related to (4) when $\mu_{0}$ and $\mu_{1}$ are nondiscrete positive Borel measures. The aim of the present contribution is the analysis of the corresponding symmetrization process when either $\mu_{0}$ or $\mu_{1}$ is a symmetric discrete measure while the other one is symmetric and continuous.

There is a set of papers dealing with the analytic properties of the sequences of orthogonal polynomials associated with (4) when $\mu_{1}$ is a discrete measure and $\mu_{0}$ is a nondiscrete measure. In (Bavinck and Meijer, 1989, 1990), the authors essentially deal with the case

$$
\mathrm{d} \mu_{0}=\chi_{[-1,1]}\left(1-x^{2}\right)^{\alpha} \mathrm{d} x+M_{0}[\delta(x+1)+\delta(x-1)] \mathrm{d} x
$$

(the Gegenbauer type measure) and

$$
\mathrm{d} \mu_{1}=M_{1}[\delta(x+1)+\delta(x-1)] \mathrm{d} x,
$$

while in (Alfaro et al., 1994) a more general situation is considered: $\mu_{0}$ is a symmetric measure and $\mathrm{d} \mu_{1}=M \delta(x) \mathrm{d} x$. These kinds of inner products are said to be Sobolev-type inner products. An extensive analysis of the recurrence relations that the sequences of corresponding orthogonal polynomials satisfy, is done in (Evans et al., 1995).

A first example of a Sobolev inner product of order 1 where $\mu_{0}$ is a discrete measure and $\mu_{1}$ is nondiscrete is considered in (Pérez and Piñar, 1996). In particular, they study Laguerre polynomials of parameter $-1, L_{n}^{(-1)}$, as a canonical example of polynomials orthogonal with respect to such a kind of inner product. Notice that they are not orthogonal with respect to a positive Borel measure. Nevertheless, they are orthogonal with respect to the Sobolev inner product given by

$$
\langle p, q\rangle_{s}=\operatorname{Mp}(0) q(0)+\int_{0}^{\infty} p^{\prime}(x) q^{\prime}(x) \mathrm{e}^{-x} \mathrm{~d} x .
$$

A natural extension of this inner product is given in (Jung et al., 1997), where some analytic properties of the sequences of polynomials orthogonal with respect to the bilinear functional

$$
\langle p, q\rangle_{s}=M p(c) q(c)+\mathbf{L}\left(p^{\prime} q^{\prime}\right)
$$

are studied. 
For a unified approach to these cases, see (Alfaro et al., 1999).

The structure of the paper is the following: In Section 3, we analyze the case when the measure $\mu_{0}$ is discrete and $\mu_{1}$ is absolutely continuous. It is proven that the sequence $\left\{P_{n}\right\}$ that satisfies (3) can be expressed in terms of another sequence $\left\{S_{n}\right\}$ in the following way: $P_{n}(x)=x S_{n-1}(x)$. First, we determine the bilinear functionals such that $\left\{S_{n}\right\}$ and $\left\{R_{n}\right\}$ are the corresponding sequences of orthogonal polynomials. Then, in the particular case when the measure $\mu_{1}$ is semiclassical, we give some explicit algebraic relations between them and we obtain some recurrence relations that they satisfy. We also apply our results to the Hermite case. In Section 4 we consider the case when $\mu_{0}$ is absolutely continuous and $\mu_{1}$ is discrete. In particular, we distinguish between the case when $\mu_{1}$ is supported at zero and $\mu_{1}$ is supported at a finite subset of the real line symmetric with respect to the origin. As an example we consider such questions for $\mathrm{d} \mu_{0}=\mathrm{e}^{-x^{4}} \mathrm{~d} x$.

\section{Symmetrized Sobolev Inner Products of Order 1}

Consider two positive Borel measures $\mu_{0}$ and $\mu_{1}$ supported on the real line such that

$$
\left|\int_{\mathbb{R}} x^{n} \mathrm{~d} \mu_{i}\right|<\infty, \quad i=0,1, n \geqslant 0 .
$$

Furthermore, assume that $\mu_{0}$ and $\mu_{1}$ are supported on subsets of the real line which are symmetric with respect to the origin so that the corresponding sequences of moments

$$
c_{n}^{(i)}=\int_{\mathbb{R}} x^{n} \mathrm{~d} \mu_{i}, \quad i=0,1,
$$

satisfy $c_{2 n+1}^{(i)}=0, i=0,1, n \geqslant 0$. We introduce the symmetrized Sobolev inner product of order 1 defined in (4). Under these conditions, if we denote by $\left\{Q_{n}\right\}$ the corresponding sequence of monic polynomials orthogonal with respect to (4), then (3) holds. We are interested in the study of the orthogonality properties of the sequences $\left\{P_{n}\right\}$ and $\left\{R_{n}\right\}$ given in (3).

In the sequel, we will analyze the particular case when $\mu_{0}$ and $\mu_{1}$ are, respectively, a discrete and a nondiscrete measure as well as the situation when $\mu_{0}$ and $\mu_{1}$ are, respectively, a nondiscrete and a discrete measure. More precisely

- We will specify the orthogonality measures for the sequences $\left\{P_{n}\right\}$ and $\left\{R_{n}\right\}$.

- We will look for explicit algebraic relations between $\left\{P_{n}\right\}$ and $\left\{R_{n}\right\}$.

- Finally, we will determine recurrence relations that such sequences satisfy.

\section{Model 1: $\mu_{0}$ Is Discrete and $\mu_{1}$ Is an Absolutely Continuous Measure}

In this section, we study the symmetrization process related to a symmetrized Sobolev inner product of order 1 such that the measure $\mu_{0}$ is discrete and $\mu_{1}$ is 
an absolutely continuous measure, i.e., $\mathrm{d} \mu_{1}=\omega(x) \mathrm{d} x$. First, we prove that the sequence of polynomials $\left\{Q_{n}\right\}$ orthogonal with respect to the symmetrized inner product can be expressed in the following way:

$$
Q_{2 n}(x)=P_{n}\left(x^{2}\right)=x^{2} S_{n-1}\left(x^{2}\right), \quad Q_{2 n+1}(x)=x R_{n}\left(x^{2}\right), \quad n \geqslant 0 .
$$

Then, we find the inner products such that $\left\{S_{n}\right\}$ and $\left\{R_{n}\right\}$ are the corresponding sequences of monic orthogonal polynomials. Afterwards, assuming that the weight function $\omega$ is semiclassical, we determine an explicit algebraic relation between the sequences $\left\{S_{n}\right\}$ and $\left\{R_{n}\right\}$ as well as certain recurrence relations that they satisfy.

Consider the inner product given in (4). Suppose that $\mu_{0}$ is a discrete measure supported at $\{0\}$ and $\mu_{1}$ is a nondiscrete measure. Therefore, the inner product we are considering is

$$
\langle p, q\rangle_{s}=\lambda p(0) q(0)+\int_{\mathbb{R}} p^{\prime} q^{\prime} \mathrm{d} \mu_{1}, \quad \lambda \in \mathbb{R}_{+} .
$$

We assume that $\mu_{1}$ is a measure supported on an interval of the real line which is symmetric with respect to the origin and such that the moments of odd order vanish, i.e., $\left\langle x^{2 n}, x^{2 m+1}\right\rangle_{s}=0$ for all $n, m \geqslant 0$. In other words, the entries $(i, j)$ of the Gram matrix associated with (5) vanish when $i+j$ is an odd integer.

Let $\left\{Q_{n}\right\}$ be the sequence of monic polynomials orthogonal with respect to (5). Observe that, from (5) we get

$$
\begin{cases}\langle 1,1\rangle_{s}=\lambda, & \\ \left\langle Q_{n}, 1\right\rangle_{s}=\lambda Q_{n}(0)=0, & \text { i.e. } Q_{n}(0)=0, n \geqslant 1, \\ \left\langle Q_{n}, Q_{m}\right\rangle_{s}=\int_{\mathbb{R}} Q_{n}^{\prime} Q_{m}^{\prime} \mathrm{d} \mu_{1}, & n, m \geqslant 1 .\end{cases}
$$

From the previous expressions, we deduce

$$
\left\langle Q_{n}, p\right\rangle_{s}=\int_{\mathbb{R}} Q_{n}^{\prime} p^{\prime} \mathrm{d} \mu_{1}, \quad n \geqslant 1, p \in \mathbb{P} .
$$

Furthermore,

$$
\begin{aligned}
& \begin{cases}Q_{2 n}(x)=P_{n}\left(x^{2}\right)=x^{2} S_{n-1}\left(x^{2}\right), & n \geqslant 1, \\
Q_{2 n+1}(x)=x R_{n}\left(x^{2}\right), & n \geqslant 0 .\end{cases} \\
& Q_{0}(x)=P_{0}(x)=1,
\end{aligned}
$$

Notice that we have introduced a new sequence of polynomials $\left\{S_{n}\right\}$ since the corresponding sequence $\left\{P_{n}\right\}$ satisfies $P_{n}(x)=x S_{n-1}(x)$.

Because of the orthogonality conditions of the sequence $\left\{Q_{n}\right\}$, for $n \neq m$, with $n, m \geqslant 1$,

$$
\begin{aligned}
0= & \left\langle Q_{2 n}, Q_{2 m}\right\rangle_{s} \\
= & 4 \int_{0}^{\infty}\left[x S_{n-1}(x) S_{m-1}(x)+\right. \\
& \left.+x^{2} S_{n-1}(x) S_{m-1}^{\prime}(x)+x^{2} S_{n-1}^{\prime}(x) S_{m-1}(x)+x^{3} S_{n-1}^{\prime}(x) S_{m-1}^{\prime}(x)\right] \mathrm{d} \hat{\mu}_{1},
\end{aligned}
$$


where $\mathrm{d} \hat{\mu}_{1}=2 \mathrm{~d} \mu_{1}\left(t^{1 / 2}\right)$. Hence, $\left\{S_{n}\right\}$ is the sequence of monic polynomials orthogonal with respect to the nondiagonal Sobolev inner product

$$
\langle p, q\rangle_{1}=4 \int_{0}^{\infty}\left[\begin{array}{ll}
p & p^{\prime}
\end{array}\right]\left[\begin{array}{cc}
1 & x \\
x & x^{2}
\end{array}\right]\left[\begin{array}{c}
q \\
q^{\prime}
\end{array}\right] x \mathrm{~d} \hat{\mu}_{1} .
$$

On the other hand, for $n \neq m$

$$
0=\left\langle Q_{2 n+1}, Q_{2 m+1}\right\rangle_{s}=\int_{0}^{\infty}\left[R_{n}(x)+2 x R_{n}^{\prime}(x)\right]\left[R_{m}(x)+2 x R_{m}^{\prime}(x)\right] \mathrm{d} \hat{\mu}_{1} .
$$

Hence, $\left\{R_{n}\right\}$ is the sequence of monic polynomials orthogonal with respect to the nondiagonal Sobolev inner product

$$
\langle p, q\rangle_{2}=\int_{0}^{\infty}\left[\begin{array}{ll}
p & p^{\prime}
\end{array}\right]\left[\begin{array}{cc}
1 & 2 x \\
2 x & 4 x^{2}
\end{array}\right]\left[\begin{array}{c}
q \\
q^{\prime}
\end{array}\right] \mathrm{d} \hat{\mu}_{1} .
$$

\subsection{EXPLICIT ALGEBRAIC RELATIONS BETWEEN $\left\{S_{n}\right\}$ AND $\left\{R_{n}\right\}$ AND RECURRENCE RELATIONS}

Since $\mu_{1}$ is an absolutely continuous measure, it can be expressed in terms of a weight function, $\mathrm{d} \mu_{1}=\omega(x) \mathrm{d} x$. The weight function $\omega$ is said to be semiclassical (Maroni, 1991; Arvesú et al., 2002) if there exist two polynomials $\phi$ and $\psi$ such that

$$
(\phi \omega)^{\prime}=\psi \omega,
$$

where $\phi$ and $\psi$ are the polynomials with minimum degree that satisfy the previous equation, $\operatorname{deg}(\phi)=k_{1} \geqslant 0, \operatorname{deg}(\psi)=k_{2}>0$, and $\phi$ is a monic polynomial. Furthermore, $\omega$ satisfies some boundary conditions, that is, $\left.\phi(x) p(x) \omega(x)\right|_{a} ^{b}=0$ for any polynomial $p$. The linear functional $\mathbf{L}$ defined as

$$
\mathbf{L}(p)=\int_{\mathbb{R}} p(x) \omega(x) \mathrm{d} x,
$$

is a semiclassical linear functional if $\omega(x)$ is a semiclassical weight function. The functional $\mathbf{L}$ is said to be of class $s$ if $s=\max \{\operatorname{deg}(\phi)-2, \operatorname{deg}(\psi)-1\}$, where $\phi$ and $\psi$ are the polynomials of minimum degree that satisfy (9).

From now on we assume that $\omega$ is a semiclassical weight function.

Next two propositions are the key to find the recurrence relations that $\left\{S_{n}\right\}$ and $\left\{R_{n}\right\}$ satisfy as well as to establish explicit algebraic relations between them.

PROPOSITION 1. If $s$ is the class of the semiclassical linear functional defined by $\omega(x)$, for $n \geqslant s+2$, we get

$$
\phi(x) Q_{n}^{\prime}(x)=n Q_{n+k_{1}-1}(x)+\sum_{j=n-s-1}^{n+k_{1}-2} \alpha_{n, j} Q_{j}(x)+\alpha_{n, 0} Q_{0}(x) .
$$


Proof. Let $\phi(x) Q_{n}^{\prime}(x)=\sum_{j=0}^{n+k_{1}-1} \alpha_{n, j} Q_{j}(x)$ be the Fourier expansion of $\phi(x) Q_{n}^{\prime}(x)$ in terms of the polynomials $\left\{Q_{n}\right\}$. Then we have

$$
\alpha_{n, j}=\frac{\left\langle\phi Q_{n}^{\prime}, Q_{j}\right\rangle_{s}}{\left\langle Q_{j}, Q_{j}\right\rangle_{s}}
$$

where

$$
\left\langle\phi Q_{n}^{\prime}, Q_{j}\right\rangle_{s}= \begin{cases}\int_{\mathbb{R}}\left(\phi^{\prime} Q_{n}^{\prime}+\phi Q{ }^{\prime \prime}\right) Q_{j}^{\prime} \mathrm{d} \mu_{1}, & \text { for } j>0 \\ \lambda \phi(0) Q_{n}^{\prime}(0), & j=0 .\end{cases}
$$

Then, for $j>0$

$$
\left\langle\phi Q_{n}^{\prime}, Q_{j}\right\rangle_{s}=\int_{\mathbb{R}} Q_{n}^{\prime} \phi^{\prime} Q_{j}^{\prime} \omega(x) \mathrm{d} x+\int_{\mathbb{R}} Q_{n}^{\prime \prime} \phi Q_{j}^{\prime} \omega(x) \mathrm{d} x .
$$

Applying integration by parts to the second integral in the previous expression and taking into account (9), we get

$$
\left\langle\phi Q_{n}^{\prime}, Q_{j}\right\rangle_{s}=-\int_{\mathbb{R}} Q_{n}^{\prime} \phi Q_{j}^{\prime \prime} \omega(x) \mathrm{d} x-\int_{\mathbb{R}} Q_{n}^{\prime} Q_{j}^{\prime}\left(\psi-\phi^{\prime}\right) \omega(x) \mathrm{d} x .
$$

The polynomial $\phi Q_{j}^{\prime \prime}$ is the derivative of a polynomial of degree $j+k_{1}-1$. Therefore, the first integral will be zero if $j<n-k_{1}+1$. In an analog way, the second integral vanishes if $j<n-s-1$, where $s+1=\max \left\{k_{1}-1, k_{2}\right\}$. Therefore, $n-k_{1}+1 \geqslant n-s-1$. As a consequence, if $1 \leqslant j<n-s-1$, then

$$
\left\langle\phi Q_{n}^{\prime}, Q_{j}\right\rangle_{s}=0 \text {. }
$$

PROPOSITION 2. If $s$ is the class of the semiclassical linear functional defined by $\mathrm{d} \mu_{1}=\omega(x) \mathrm{d} x$, for $n \geqslant s+2$, then we get

$$
x \phi(x) Q_{n}^{\prime}(x)=n Q_{n+k_{1}}(x)+\sum_{j=n-s-2}^{n+k_{1}-1} \alpha_{n, j} Q_{j}(x) .
$$

Proof.

$$
\begin{aligned}
\left\langle x \phi Q_{n}^{\prime}, Q_{j}\right\rangle_{s}= & \int_{\mathbb{R}}\left[x \phi Q_{n}^{\prime}\right]^{\prime} Q_{j}^{\prime} \omega(x) \mathrm{d} x \\
= & \int_{\mathbb{R}} \phi Q_{n}^{\prime} Q_{j}^{\prime} \omega(x) \mathrm{d} x+\int_{\mathbb{R}} x \phi^{\prime} Q_{n}^{\prime} Q_{j}^{\prime} \omega(x) \mathrm{d} x+ \\
& +\int_{\mathbb{R}} x \phi Q_{n}^{\prime \prime} Q_{j}^{\prime} \omega(x) \mathrm{d} x
\end{aligned}
$$

applying integration by parts to the third integral, we obtain

$$
=-\int_{\mathbb{R}}\left[x \phi Q_{n}^{\prime} Q_{j}^{\prime \prime}+x Q_{n}^{\prime} Q_{j}^{\prime}\left(\psi-\phi^{\prime}\right)\right] \omega(x) \mathrm{d} x .
$$


Similar arguments to those given in the proof of Proposition 1 lead us to deduce that the previous integral vanishes if $j<n-s-2$, which proves the proposition.

Now we introduce a lemma from a previous paper that will be very useful to prove Proposition 4 and Proposition 5.

LEMMA 3 (Bueno and Marcellán, 2003). Let $\mathbf{L}$ be a symmetric semiclassical linear functional satisfying $D(\phi \mathbf{L})=\psi \mathbf{L}$. If s denotes the class of $\mathbf{L}$, then

(1) If $s$ is an even number, then $\phi$ is an even function.

(2) If $s$ is an odd number, then $\phi$ is an odd function.

In the sequel, when $s$ is an even number, we write $s=2 r, k_{1}=2 k$, and $\phi(x)=$ $\tilde{\phi}\left(x^{2}\right)$. When $s$ is odd, we write $s=2 r+1, k_{1}=2 k+1$, and $\phi(x)=x \hat{\phi}\left(x^{2}\right)$.

PROPOSITION 4. Consider a symmetrized Sobolev inner product as in (5) and suppose that $\mathrm{d} \mu_{1}=\omega(x) \mathrm{d} x$ is an absolutely continuous semiclassical measure that satisfies (9). Let $\left\{Q_{n}\right\}$ be the sequence of monic polynomials orthogonal with respect to (5). Assume that $\left\{S_{n}\right\}$ and $\left\{R_{n}\right\}$ are the sequences such that (6) holds. If $s$ denotes the class of the semiclassical linear functional defined by $\omega$, then, the following algebraic relations between $\left\{S_{n}\right\}$ and $\left\{R_{n}\right\}$ take place.

(1) If $s$ is even, then $\phi(x)=\tilde{\phi}\left(x^{2}\right)$ and

$$
\begin{aligned}
& 2 \tilde{\phi}(x)\left[S_{m-1}(x)+x S_{m-1}^{\prime}(x)\right] \\
& \quad=2 m R_{m+k-1}(x)+\sum_{j=m-r-1}^{m+k-2} \alpha_{2 m, 2 j+1} R_{j}(x), \\
& \tilde{\phi}(x)\left[R_{m}(x)+2 x R_{m}^{\prime}(x)\right] \\
& =(2 m+1) x S_{m+k-1}(x)+\sum_{j=m-r-1}^{m+k-2} \alpha_{2 m+1,2 j+2} x S_{j}(x)+\alpha_{2 m+1,0} .
\end{aligned}
$$

(2) If $s$ is odd, then $\phi(x)=x \hat{\phi}\left(x^{2}\right)$ and

$$
\begin{aligned}
& 2 \hat{\phi}(x)\left[x S_{m-1}(x)+x^{2} S_{m-1}^{\prime}(x)\right] \\
& \quad=2 m R_{m+k}(x)+\sum_{j=m-r-2}^{m+k-1} \alpha_{2 m, 2 j+1} R_{j}(x), \\
& \hat{\phi}(x)\left[R_{m}(x)+2 x R_{m}^{\prime}(x)\right] \\
& \quad=(2 m+1) S_{m+k}(x)+\sum_{j=m-r-2}^{m+k-1} \alpha_{2 m+1,2 j+2} S_{j}(x) .
\end{aligned}
$$


Proof. For $n=2 m,(10)$ becomes

$$
\phi(x) Q_{2 m}^{\prime}(x)=2 m Q_{2 m+k_{1}-1}(x)+\sum_{j=2 m-s-1}^{2 m+k_{1}-2} \alpha_{2 m, j} Q_{j}(x)+\alpha_{2 m, 0} .
$$

Assume that the class $s$ of the semiclassical functional associated with $\omega$ is even. From Lemma $3, \phi$ is an even function and $\phi(x)=\tilde{\phi}\left(x^{2}\right)$. Moreover, we write $k_{1}=2 k$ and $s=2 r$. In such a case, taking into account (6), the expression (16) can be written in the following way

$$
\begin{aligned}
& 2 x \tilde{\phi}\left(x^{2}\right)\left[S_{m-1}\left(x^{2}\right)+x^{2} S_{m-1}^{\prime}\left(x^{2}\right)\right] \\
& \quad=2 m R_{m+k-1}+\sum_{i=m-r-1}^{m+k-2} \alpha_{2 m, 2 j+1} x R_{j}\left(x^{2}\right)
\end{aligned}
$$

and the result in (12) follows.

If we rewrite (10) for $n=2 m+1$, (13) can be obtained in a similar way.

The previous reasoning is also valid to deduce the algebraic relations in (14) and (15) if (11) is considered instead of (10).

PROPOSITION 5. Consider a symmetrized Sobolev inner product as in (5) and suppose that $\mathrm{d} \mu_{1}=\omega(x) \mathrm{d} x$ is an absolutely continuous semiclassical measure that satisfies (9). Let $\left\{Q_{n}\right\}$ be the sequence of monic polynomials orthogonal with respect to (5). Assume that $\left\{S_{n}\right\}$ and $\left\{R_{n}\right\}$ are the sequences such that (6) holds. If $s$ denotes the class of the semiclassical linear functional defined by $\omega$, then, the following recurrence relations for $\left\{S_{n}\right\}$ and $\left\{R_{n}\right\}$ are obtained.

(1) If $s$ is even, then $\phi(x)=\tilde{\phi}\left(x^{2}\right)$ and

$$
\begin{aligned}
& 2 \tilde{\phi}(x)\left[S_{m-1}(x)+x S_{m-1}^{\prime}(x)\right] \\
& \quad=2 m S_{m+k-1}(x)+\sum_{j=m-r-2}^{m+k-2} \alpha_{2 m, 2 j+2} S_{j}(x), \\
& \tilde{\phi}(x)\left[R_{m}(x)+2 x R_{m}^{\prime}(x)\right] \\
& \quad=(2 m+1) R_{m+k}(x)+\sum_{j=m-r-1}^{m+k-1} \alpha_{2 m+1,2 j+1} R_{j}(x) .
\end{aligned}
$$

(2) If $s$ is odd, then $\phi(x)=x \hat{\phi}\left(x^{2}\right)$ and

$$
\begin{aligned}
& 2 \hat{\phi}(x)\left[S_{m-1}(x)+x S_{m-1}^{\prime}(x)\right] \\
& =2 m S_{m+k-1}(x)+\sum_{j=m-r-2}^{m+k-2} \alpha_{2 m, 2 j+2} S_{j}(x)+\alpha_{2 m, 0},
\end{aligned}
$$




$$
\begin{aligned}
& \hat{\phi}(x)\left[R_{m}(x)+2 x R_{m}^{\prime}(x)\right] \\
& =(2 m+1) R_{m+k}(x)+\sum_{j=m-r-1}^{m+k-1} \alpha_{2 m+1,2 j+1} R_{j}(x) .
\end{aligned}
$$

Proof. For $n=2 m$, (11) becomes

$$
x \phi(x) Q_{2 m}^{\prime}(x)=2 m Q_{2 m+k_{1}}(x)+\sum_{j=2 m-s-2}^{2 m+k_{1}-1} \alpha_{2 m, j} Q_{j}(x) .
$$

Assume that $s$ is even. Then, from Lemma 3, $\phi$ is an even function and $\phi(x)=$ $\tilde{\phi}\left(x^{2}\right)$. Taking into account (6), the expression (21) can be written in the following way

$$
\begin{aligned}
& 2 x^{2} \tilde{\phi}\left(x^{2}\right)\left[S_{m-1}\left(x^{2}\right)+x^{2} S_{m-1}^{\prime}\left(x^{2}\right)\right] \\
& \quad=2 m x^{2} S_{m+k-1}\left(x^{2}\right)+\sum_{j=m-r-2}^{m+k-2} \alpha_{2 m, 2 j} x^{2} S_{j}\left(x^{2}\right),
\end{aligned}
$$

and the result in (17) follows. The remaining recurrence relations are obtained in a similar way.

\subsection{THE HERMITE CASE}

It has been proven that, if $\left\{Q_{n}\right\}$ is the sequence of monic polynomials orthogonal with respect to (5), then

$$
\left\langle Q_{n}, Q_{m}\right\rangle_{s}=\int_{\mathbb{R}} Q_{n}^{\prime} Q_{m}^{\prime} \mathrm{d} \mu_{1}, \quad \text { for } n+m \geqslant 1 .
$$

Therefore, $\left\{Q_{n}^{\prime}\right\}_{n=1}^{\infty}$ is a sequence of polynomials orthogonal with respect to the inner product

$$
\begin{aligned}
&\qquad p, q\rangle=\int_{\mathbb{R}} p q \mathrm{~d} \mu_{1} . \\
& \text { If } \mathrm{d} \mu_{1}=\mathrm{e}^{-x^{2}} \mathrm{~d} x \text { then } \\
& Q_{n}^{\prime}(x)=n H_{n-1}(x), \quad n \geqslant 1,
\end{aligned}
$$

where $H_{n}(x)$ denotes the $n$th monic Hermite polynomial. In such a case and taking into account that $Q_{n}(0)=0$ for $n \geqslant 1$, we get

$$
\frac{Q_{n}(x)}{n}=\int_{0}^{x} H_{n-1}(t) \mathrm{d} t .
$$


Applying integration by parts in the previous integral and taking into account that $H_{n}^{\prime}(x)=n H_{n-1}(x)$,

$$
\frac{Q_{n}(x)}{n}=x H_{n-1}(x)-\int_{0}^{x}(n-1) t H_{n-2}(t) \mathrm{d} t .
$$

From the three-term recurrence relation for Hermite polynomials (Chihara, 1978), we get

$$
\begin{aligned}
\frac{Q_{n}(x)}{n} & =x H_{n-1}(x)-\int_{0}^{x}(n-1)\left[H_{n-1}(t)+\frac{n-2}{2} H_{n-3}(t)\right] \mathrm{d} t \\
& =x H_{n-1}(x)-(n-1) \frac{Q_{n}(x)}{n}-\frac{n-1}{2} Q_{n-2}(x) .
\end{aligned}
$$

Hence,

$$
x H_{n-1}(x)=Q_{n}(x)+\frac{n-1}{2} Q_{n-2}, \quad n \geqslant 3 .
$$

Applying the three-term recurrence relation again

$$
H_{n}(x)+\frac{n-1}{2} H_{n-2}(x)=Q_{n}(x)+\frac{n-1}{2} Q_{n-2}(x), \quad n \geqslant 3 .
$$

Notice that this expression has the same structure as in the case of symmetric coherent pairs (Kim et al., 2002; Meijer, 1997), but this concept has a sense only for the continuous case.

On the other hand, from (22)

$$
Q_{n}(x)=H_{n}(x)-H_{n}(0),
$$

i.e.,

$$
Q_{2 n}(x)=H_{2 n}(x)-H_{2 n}(0), \quad Q_{2 n+1}(x)=H_{2 n+1}(x) .
$$

Thus, since (6) holds, and taking into account that $H_{2 m}(x)=L_{m}^{(-1 / 2)}\left(x^{2}\right)$, and $H_{2 m+1}(x)=x L_{m}^{(1 / 2)}\left(x^{2}\right)$, we deduce

$$
\begin{aligned}
& P_{n}(x)=x S_{n-1}(x)=L_{n}^{(-1 / 2)}(x)-L_{n}^{(-1 / 2)}(0), \\
& R_{n}(x)=L_{n}^{(1 / 2)}(x) .
\end{aligned}
$$

\subsubsection{Orthogonality Measures for $\left\{S_{n}\right\}$ and $\left\{R_{n}\right\}$}

According to (7), $\left\{S_{n}\right\}$ is orthogonal with respect to the nondiagonal Sobolev inner product

$$
\langle p, q\rangle_{1}=4 \int_{0}^{\infty}\left[\begin{array}{ll}
p & p^{\prime}
\end{array}\right]\left[\begin{array}{cc}
1 & t \\
t & t^{2}
\end{array}\right]\left[\begin{array}{c}
q \\
q^{\prime}
\end{array}\right] t^{1 / 2} \mathrm{e}^{-t} \mathrm{~d} t .
$$


Nevertheless, applying integration by parts, the previous inner product can be reduced to a diagonal form although the measure involved in the standard part is a signed measure.

$$
\langle p, q\rangle_{1}=4 \int_{0}^{\infty}\left[\begin{array}{ll}
p & p^{\prime}
\end{array}\right]\left[\begin{array}{cc}
t-1 / 2 & 0 \\
0 & t^{2}
\end{array}\right]\left[\begin{array}{c}
q \\
q^{\prime}
\end{array}\right] t^{1 / 2} \mathrm{e}^{-t} \mathrm{~d} t .
$$

From (8), $\left\{R_{n}\right\}$ is orthogonal with respect to the nondiagonal Sobolev inner product

$$
\langle p, q\rangle_{2}=\int_{0}^{\infty}\left[\begin{array}{ll}
p & p^{\prime}
\end{array}\right]\left[\begin{array}{cc}
1 & 2 t \\
2 t & 4 t^{2}
\end{array}\right]\left[\begin{array}{c}
q \\
q^{\prime}
\end{array}\right] t^{-1 / 2} \mathrm{e}^{-t} \mathrm{~d} t .
$$

Again, taking into account an integration by parts, the inner product $\langle,\rangle_{2}$ can be given by

$$
\langle p, q\rangle_{2}=\int_{0}^{\infty}\left[\begin{array}{ll}
p & p^{\prime}
\end{array}\right]\left[\begin{array}{cc}
2 & 0 \\
0 & 4 t
\end{array}\right]\left[\begin{array}{c}
q \\
q^{\prime}
\end{array}\right] t^{1 / 2} \mathrm{e}^{-t} \mathrm{~d} t,
$$

that is, it can be reduced to a diagonal form.

\subsubsection{Recurrence Relations and Explicit Algebraic Relations}

Since $\left\{R_{n}\right\}$ is the sequence of Laguerre polynomials of parameter $1 / 2$, we only deduce recurrence relations for the sequences $\left\{S_{n}\right\}$ and $\left\{Q_{n}\right\}$. We also deduce two explicit algebraic relations between the sequences $\left\{R_{n}\right\}$ and $\left\{S_{n}\right\}$.

Consider the equation given in (23) for $n=2 m$. Then, taking into account (6), we obtain

$$
L_{n}^{(1 / 2)}(x)=S_{n}(x)+\frac{2 n+1}{2} S_{n-1}(x), \quad n \geqslant 1 .
$$

The three-term recurrence relation that Laguerre polynomials of parameter $1 / 2$ satisfy (Chihara, 1978) is

$$
\begin{aligned}
& L_{n}^{(1 / 2)}(x)=\left(x-2 n+\frac{1}{2}\right) L_{n-1}^{(1 / 2)}(x)-(n-1)\left(n-\frac{1}{2}\right) L_{n-2}^{(1 / 2)}(x), \\
& \quad n \geqslant 2 .
\end{aligned}
$$

Substituting (26) in (27) and simplifying the result, we deduce a four-term recurrence relation for $\left\{S_{n}\right\}$.

$$
\begin{aligned}
S_{n}(x)= & (x-3 n) S_{n-1}(x)+ \\
& +\left(n-\frac{1}{2}\right)\left[x-3\left(n-\frac{1}{2}\right)\right] S_{n-2}(x)- \\
& -(n-1)\left(n-\frac{1}{2}\right)\left(n-\frac{3}{2}\right) S_{n-3}(x), \quad n \geqslant 3 .
\end{aligned}
$$


We deduce now the recurrence relation that the polynomials $\left\{Q_{n}\right\}$ satisfy. Taking into account the three-term recurrence relation that Hermite polynomials satisfy

$$
H_{n}(x)=x H_{n-1}(x)-\frac{1}{2}(n-1) H_{n-2}(x), \quad n \geqslant 2,
$$

and plugging the expression (23) in it, we obtain a five-term recurrence relation for $\left\{Q_{n}\right\}$.

$$
\begin{aligned}
Q_{n+1}(x)= & x Q_{n}(x)+\left(\frac{1}{2}-n\right) Q_{n-1}(x)+ \\
& +\left(\frac{n-1}{2}\right) x Q_{n-2}(x)-\frac{(n-1)(n-2)}{4} Q_{n-3}(x), \\
n \geqslant 3 . &
\end{aligned}
$$

Finally, we deduce explicit algebraic relations between the sequences $\left\{S_{n}\right\}$ and $\left\{R_{n}\right\}$. Notice that (26) can be rewritten in the following way

$$
R_{n}(x)=S_{n}(x)+\frac{2 n+1}{2} S_{n-1}(x), \quad n \geqslant 1,
$$

which gives us an explicit algebraic relation between the two sequences in consideration.

On the other hand, expressing (29) for $n=2 m+1$, and from (6) we get

$$
\begin{aligned}
& S_{m}(x)+\left(2 m+\frac{1}{2}\right) S_{m-1}(x)+\frac{m(2 m-1)}{2} S_{m-2}(x) \\
& \quad=R_{m}(x)+m R_{m-1}(x), \quad m \geqslant 1,
\end{aligned}
$$

or, equivalently,

$$
\begin{aligned}
& S_{m}(x)+\left(2 m+\frac{1}{2}\right) S_{m-1}(x)+\frac{m(2 m-1)}{2} S_{m-2}(x) \\
& \quad=L_{m}^{(1 / 2)}(x)+m L_{m-1}^{(1 / 2)}(x), \quad m \geqslant 1 .
\end{aligned}
$$

\section{Model 2: $\mu_{0}$ Absolutely Continuous and $\mu_{1}$ Discrete}

Next we deal with the study of the symmetrization process related to symmetrized Sobolev inner products of order 1

$$
\langle p, q\rangle_{s}=\int_{\mathbb{R}} p q \mathrm{~d} \mu_{0}+\int_{\mathbb{R}} p^{\prime} q^{\prime} \mathrm{d} \mu_{1},
$$

where $\mu_{1}$ is a discrete measure and $\mu_{0}$ is a nondiscrete positive Borel measure. We consider two different situations. First, we assume that $\mu_{1}$ is supported at zero. Secondly, we study the general case when $\mu_{1}$ is supported on a finite subset of the real line symmetric with respect to the origin containing more than one point. 


\section{1. $\mu_{1}$ IS SUPPORTED AT ZERO}

We first analyze the case when $\mu_{1}$ is supported at $\{0\}$. Consider the product

$$
\langle p, q\rangle_{s}=\int_{\mathbb{R}} p q \mathrm{~d} \mu_{0}+\lambda p^{\prime}(0) q^{\prime}(0),
$$

where $\lambda$ is a positive real number. We assume that $\mu_{0}$ is a nondiscrete measure supported on a subset of the real line symmetric with respect to the origin, and such that the corresponding odd moments vanish, i.e.,

$$
c_{2 n+1}=\int_{\mathbb{R}} x^{2 n+1} \mathrm{~d} \mu_{0}=0, \quad \text { for all } n \geqslant 0 .
$$

Such a kind of inner products are called Sobolev type inner products (Alfaro et al., 1994).

\subsubsection{Orthogonality Measures for $\left\{P_{n}\right\}$ and $\left\{R_{n}\right\}$}

Let $\left\{Q_{n}\right\}$ be the sequence of monic polynomials orthogonal with respect to (32). Then, (3) holds for certain sequences of monic polynomials $\left\{P_{n}\right\}$ and $\left\{R_{n}\right\}$. For $n \neq m$,

$$
0=\left\langle Q_{2 n}, Q_{2 m}\right\rangle_{s}=2 \int_{0}^{\infty} P_{n}(x) P_{m}(x) \mathrm{d} \mu_{0}\left(x^{1 / 2}\right) .
$$

Hence, $\left\{P_{n}\right\}$ is the sequence of monic polynomials orthogonal with respect to the standard inner product

$$
\langle p, q\rangle_{1}=\int_{0}^{\infty} p q \mathrm{~d} \hat{\mu}_{0},
$$

where $\mathrm{d} \hat{\mu}_{0}=2 \mathrm{~d} \mu_{0}\left(x^{1 / 2}\right)$. On the other hand, if $n \neq m$, then

$$
0=\left\langle Q_{2 n+1}, Q_{2 m+1}\right\rangle_{s}=2 \int_{0}^{\infty} x R_{n}(x) R_{m}(x) \mathrm{d} \mu_{0}\left(x^{1 / 2}\right)+\lambda R_{n}(0) R_{m}(0) .
$$

Thus $\left\{R_{n}\right\}$ is the sequence of monic polynomials orthogonal with respect to the standard inner product

$$
\langle p, q\rangle_{2}=\int_{0}^{\infty} x p q \mathrm{~d} \hat{\mu}_{0}+\lambda p(0) q(0) .
$$

\subsubsection{Explicit Algebraic Relations between $\left\{P_{n}\right\}$ and $\left\{R_{n}\right\}$}

In this case, Proposition 7 gives an algebraic relation between the sequences $\left\{P_{n}\right\}$ and $\left\{R_{n}\right\}$. Notice that the polynomial $R_{n}$ is expressed in terms of the polynomials $P_{n}^{*}$ and $P_{n-1}^{* *}$, i.e., in terms of the sequences of the monic kernel polynomials 
with parameter 0 associated with $\left\{P_{n}\right\}$ and $\left\{P_{n}^{*}\right\}$, respectively. However, taking into account (2), it is straightforward to obtain the explicit algebraic relation we were looking for.

Consider now the standard inner product

$$
\langle p, q\rangle=\int_{\mathbb{R}} p q \mathrm{~d} \mu_{0},
$$

and let $\left\{T_{n}\right\}$ be the corresponding sequence of monic orthogonal polynomials. Then, the following relation holds.

PROPOSITION 6. Assume that $\left\{Q_{n}\right\}$ is the sequence of monic polynomials orthogonal with respect to (32), $\left\{P_{n}\right\}$ is the sequence of polynomials such that $Q_{2 n}(x)$ $=P_{n}\left(x^{2}\right),\left\{P_{n}^{*}\right\}$ is the sequence of monic kernel polynomials with parameter 0 associated with $\left\{P_{n}\right\}$, and $\left\{T_{n}\right\}$ is the sequence of monic polynomials orthogonal with respect to the inner product given in (35), then

$$
T_{2 n}(x)=P_{n}\left(x^{2}\right)=Q_{2 n}(x), \quad T_{2 n+1}(x)=x P_{n}^{*}\left(x^{2}\right), \quad n \geqslant 0 .
$$

Proof. Let us take into account the Fourier expansion of $Q_{n}$ in terms of $\left\{T_{n}\right\}$

$$
Q_{n}(x)=T_{n}(x)+\sum_{j=0}^{n-1} \alpha_{n j} T_{j}(x), \quad n \geqslant 1 .
$$

Then, for $0 \leqslant j<n$, and from (32)

$$
\alpha_{n, j}=\frac{\left\langle Q_{n}, T_{j}\right\rangle}{\left\langle T_{j}, T_{j}\right\rangle}=\frac{\int_{\mathbb{R}} Q_{n} T_{j} \mathrm{~d} \mu_{0}}{\left\|T_{j}\right\|^{2}}=\frac{-\lambda Q_{n}^{\prime}(0) T_{j}^{\prime}(0)}{\left\|T_{j}\right\|^{2}} .
$$

Plugging the previous expression in (37) we obtain

$$
Q_{n}(x)=T_{n}(x)-\sum_{j=0}^{n-1} \frac{\lambda Q_{n}^{\prime}(0) T_{j}^{\prime}(0)}{\left\|T_{j}\right\|^{2}} T_{j}(x), \quad n \geqslant 1 .
$$

Since $Q_{2 m}(x)=P_{m}\left(x^{2}\right), Q_{2 m}^{\prime}(0)=0$. Thus, for $n=2 m$, (38) becomes

$$
Q_{2 m}(x)=T_{2 m}(x), \quad m \geqslant 0,
$$

or, equivalently,

$$
T_{2 m}(x)=P_{m}\left(x^{2}\right), \quad m \geqslant 0 .
$$

Moreover, taking into account that (35) is a standard inner product

$$
T_{2 m+1}(x)=x P_{m}^{*}\left(x^{2}\right), \quad m \geqslant 0 .
$$


PROPOSITION 7. Let $\left\{Q_{n}\right\}$ be the sequence of monic polynomials orthogonal with respect to (32). If $\left\{P_{n}\right\}$ and $\left\{R_{n}\right\}$ are the sequences of polynomials such that (3) is satisfied, then the following relations hold.

$$
\begin{aligned}
& R_{0}(x)=P_{0}^{*}(x), \quad R_{n}(x)=P_{n}^{*}(x)+\alpha_{n} P_{n-1}^{* *}(x), \quad n \geqslant 1, \\
& \alpha_{n}=-\frac{\lambda R_{n}(0) P_{n-1}^{*}(0)}{\left\|P_{n-1}^{*}\right\|_{*}^{2}},
\end{aligned}
$$

with $\left\|P_{n}^{*}\right\|_{*}=\left\|x P_{n}^{*}\right\|$. Moreover, $\left\{P_{n}^{* *}\right\}$ denotes the sequence of kernel polynomials with parameter 0 associated with $\left\{P_{n}^{*}\right\}$.

Proof. Put $n=2 m+1$ in (38) to obtain

$$
Q_{2 m+1}(x)=T_{2 m+1}(x)-\sum_{j=0}^{2 m} \frac{\lambda Q_{2 m+1}^{\prime}(0) T_{j}^{\prime}(0)}{\left\|T_{j}\right\|^{2}} T_{j}(x), \quad m \geqslant 0 .
$$

But $Q_{2 m+1}^{\prime}(x)=R_{m}\left(x^{2}\right)+2 x^{2} R_{m}^{\prime}\left(x^{2}\right)$, hence we deduce

$$
Q_{2 m+1}^{\prime}(0)=R_{m}(0), \quad m \geqslant 0 .
$$

On the other hand, from Proposition 6

$$
\begin{aligned}
& T_{2 j}^{\prime}(x)=2 x P_{j}^{\prime}\left(x^{2}\right), \quad j \geqslant 1, \\
& T_{2 j+1}^{\prime}(x)=P_{j}^{*}\left(x^{2}\right)+2 x^{2}\left(P_{j}^{*}\right)^{\prime}\left(x^{2}\right), \quad j \geqslant 0,
\end{aligned}
$$

and hence

$$
T_{j}^{\prime}(0)= \begin{cases}0 & \text { if } j \text { is even, } \\ P_{\frac{j-1}{2}}^{*}(0) & \text { if } j \text { is odd. }\end{cases}
$$

From (40) we get

$$
R_{m}\left(x^{2}\right)=P_{m}^{*}\left(x^{2}\right)-R_{m}(0) \sum_{j=0}^{m-1} \frac{\lambda P_{j}^{*}(0) P_{j}^{*}\left(x^{2}\right)}{\left\|x P_{j}^{*}\left(x^{2}\right)\right\|^{2}}, \quad m \geqslant 1 .
$$

Recall that, given a sequence of polynomials $\left\{V_{n}\right\}$, the sequence $\left\{V_{n}^{*}\right\}$ of monic kernel polynomials with parameter 0 associated with $\left\{V_{n}\right\}$ satisfies

$$
\frac{V_{n}(0) V_{n}^{*}(x)}{\left\|V_{n}\right\|^{2}}=\sum_{k=0}^{n} \frac{V_{k}(x) V_{k}(0)}{\left\|V_{k}\right\|^{2}}, \quad n \geqslant 0 .
$$

Then, taking into account the previous definition

$$
R_{m}(x)=P_{m}^{*}(x)-\frac{\lambda R_{m}(0) P_{m-1}^{*}(0)}{\left\|P_{m-1}^{*}\right\|_{*}^{2}} P_{m-1}^{* *}(x), \quad m \geqslant 1,
$$

and the result follows. 


\subsubsection{Recurrence Relations}

In this subsection, we give the three-term recurrence relations that the sequences $\left\{Q_{n}\right\},\left\{P_{n}\right\}$, and $\left\{R_{n}\right\}$ satisfy.

PROPOSITION 8. Consider a symmetrized Sobolev inner product as in (32). Let $\left\{Q_{n}\right\}$ be the corresponding sequence of monic orthogonal polynomials. Assume that $\left\{S_{n}\right\}$ and $\left\{R_{n}\right\}$ are the sequences such that (6) holds. Then, the following recurrence relations are obtained.

$$
\begin{aligned}
& Q_{n+2}(x)=\left(x^{2}-\beta_{n, n}\right) Q_{n}(x)-\beta_{n, n-2} Q_{n-2}(x), \quad n \geqslant 2, \\
& P_{n+1}(x)=\left(x-\beta_{2 n, 2 n}\right) P_{n}(x)-\beta_{2 n, 2 n-2} P_{n-1}(x), \quad n \geqslant 1, \\
& R_{n+1}(x)=\left(x-\beta_{2 n+1,2 n+1}\right) R_{n}(x)-\beta_{2 n+1,2 n-1} R_{n-1}(x), \quad n \geqslant 1 .
\end{aligned}
$$

Proof. The multiplication by $x^{2}$ is a symmetric operator with respect to the inner product (32), i.e.,

$$
\left\langle x^{2} Q_{n}, Q_{j}\right\rangle_{s}=\left\langle Q_{n}, x^{2} Q_{j}\right\rangle_{s} .
$$

Furthermore, $\left\langle x^{2} Q_{n}, Q_{j}\right\rangle_{s}=0$, for $0 \leqslant j<n-2$, and, as a consequence,

$$
x^{2} Q_{n}(x)=Q_{n+2}(x)+\beta_{n, n} Q_{n}(x)+\beta_{n, n-2} Q_{n-2}(x), \quad n \geqslant 1 .
$$

From (41), for $n=2 m$ and $n=2 m+1$, respectively, the recurrence relations for $\left\{P_{n}\right\}$ and $\left\{R_{n}\right\}$ are obtained.

\subsubsection{Particular Case: $\mathrm{d} \mu_{0}=\mathrm{e}^{-x^{4}} \mathrm{~d} x$}

Consider the inner product

$$
\langle p, q\rangle_{s}=\int_{\mathbb{R}} p q \mathrm{e}^{-x^{4}} \mathrm{~d} x+\lambda p^{\prime}(0) q^{\prime}(0) .
$$

Let $\left\{Q_{n}\right\}$ be the sequence of monic polynomials orthogonal with respect to (44). Consider the sequences $\left\{P_{n}\right\}$ and $\left\{R_{n}\right\}$ such that (3) holds.

From (33), $\left\{P_{n}\right\}$ is the sequence of monic polynomials orthogonal with respect to the standard inner product

$$
\langle p, q\rangle_{1}=\int_{0}^{\infty} p q x^{-1 / 2} \mathrm{e}^{-x^{2}} \mathrm{~d} x .
$$

Similarly, from (34), $\left\{R_{n}\right\}$ is the sequence of monic polynomials orthogonal with respect to the standard inner product

$$
\langle p, q\rangle_{2}=\int_{0}^{\infty} p q x^{1 / 2} \mathrm{e}^{-x^{2}} \mathrm{~d} x+\lambda p(0) q(0) .
$$


Taking into account (2) and Proposition 7, an explicit algebraic relation between $\left\{P_{n}\right\}$ and $\left\{R_{n}\right\}$ is obtained.

$$
\begin{aligned}
x^{2} R_{n}(x)= & \left(x+\alpha_{n}\right) P_{n+1}(x)-\left[\left(x+\alpha_{n}\right) \frac{P_{n+1}(0)}{P_{n}(0)}+\frac{P_{n}^{*}(0)}{P_{n-1}^{*}(0)} \alpha_{n}\right] P_{n}(x)+ \\
& +\frac{P_{n}^{*}(0) P_{n}(0)}{P_{n-1}^{*}(0) P_{n-1}(0)} \alpha_{n} P_{n-1}(x), \quad n \geqslant 1,
\end{aligned}
$$

where $\alpha_{n}$ is given in (39). Since $\left\{P_{n}\right\}$ and $\left\{R_{n}\right\}$ are orthogonal with respect to standard inner products, they satisfy three-term recurrence relations whose parameters can be calculated by Stieltjes formulas (Gautschi, 1982).

Finally, we express the parameters in the recurrence relation that the sequence $\left\{Q_{n}\right\}$ satisfies in terms of the parameters of the three-term recurrence relation that Freud polynomials satisfy.

Let us consider the standard inner product

$$
\langle p, q\rangle=\int_{\mathbb{R}} p q \mathrm{e}^{-x^{4}} \mathrm{~d} x .
$$

Let $\left\{F_{n}\right\}$ be the sequence of monic polynomials orthogonal with respect to (47). It is a basic example of Freud Polynomials. It is well known (Nevai, 1983, 1984; Cachafeiro et al., 2003) that $\left\{F_{n}\right\}$ satisfies a three-term recurrence relation

$$
F_{n+1}(x)=x F_{n}(x)-c_{n} F_{n-1}(x), \quad n \geqslant 1,
$$

with initial conditions

$$
F_{0}(x)=1, \quad F_{1}(x)=x,
$$

and

$$
c_{0}=0, \quad c_{1}=\frac{\Gamma(3 / 4)}{\Gamma(1 / 4)}, \quad n=4 c_{n}\left(c_{n+1}+c_{n}+c_{n-1}\right), \quad n \geqslant 1 .
$$

For $n=2 m-1$, (48) becomes

$$
F_{2 m}(x)=x F_{2 m-1}(x)-c_{2 m-1} F_{2 m-2}(x), \quad m \geqslant 1 .
$$

Taking into account (36), we get

$$
P_{m}(x)=x P_{m-1}^{*}(x)-c_{2 m-1} P_{m-1}(x), \quad m \geqslant 1 .
$$

From (2), we obtain

$$
c_{2 m-1}=-\frac{P_{m}(0)}{P_{m-1}(0)}, \quad m \geqslant 1 .
$$

For $n=2 m$, (48) becomes

$$
F_{2 m+1}(x)=x F_{2 m}(x)-c_{2 m} F_{2 m-1}(x), \quad m \geqslant 1 .
$$


Again from (36), we get

$$
P_{m}^{*}(x)=P_{m}(x)-c_{2 m} P_{m-1}^{*}(x), \quad m \geqslant 1 .
$$

From (2) and (49), we obtain

$$
P_{m+1}(x)=\left[x-c_{2 m+1}-c_{2 m}\right] P_{m}(x)-c_{2 m} c_{2 m-1} P_{m-1}(x), \quad m \geqslant 1 .
$$

Finally, taking into account (42), from (49) and (50) we get

$$
\beta_{2 m, 2 m}=c_{2 m}+c_{2 m+1}, \quad \beta_{2 m, 2 m-2}=c_{2 m} c_{2 m-1}, \quad m \geqslant 1 .
$$

Assume that $\left\{\xi_{m}\right\}$ and $\left\{\gamma_{m}\right\}$ are the sequences of parameters of the three-term recurrence relation that the sequence $\left\{P_{m}^{*}\right\}$ satisfies, i.e.,

$$
P_{m+1}^{*}(x)=\left(x-\xi_{m}\right) P_{m}^{*}(x)-\gamma_{m} P_{m-1}^{*}(x), \quad m \geqslant 1 .
$$

Taking into account the definition of kernel polynomials, (50), and (49) we get

$$
\begin{aligned}
& \xi_{m}=c_{2 m+1}+c_{2 m+2}, \quad m \geqslant 0, \\
& \gamma_{m}=c_{2 m} c_{2 m+1}, \quad m \geqslant 1 .
\end{aligned}
$$

On the other hand, we know that $\left\{R_{n}\right\}$ satisfies the three-term recurrence relation given in (43). Taking into account Proposition 7 and (52), we get

$$
\begin{aligned}
& \beta_{1,1}=c_{1}+c_{2}-\alpha_{1}, \\
& \beta_{2 m+1,2 m+1}=\alpha_{m}-\alpha_{m+1}+c_{2 m+1}+c_{2 m+2}, \quad m \geqslant 1, \\
& \beta_{3,1}=c_{2}+c_{3}+\alpha_{1}+\alpha_{2}\left[c_{3}+c_{4}-\frac{P_{2}^{*}(0)}{P_{1}^{*}(0)}\right], \\
& \beta_{2 m+1,2 m-1}=\frac{c_{2 m-1} c_{2 m-2} P_{m-2}^{*}(0)}{\alpha_{m-1} P_{m-1}^{*}(0)}\left[\alpha_{m+1}\left(c_{2 m+1}+c_{2 m+2}+\frac{P_{m+1}^{*}(0)}{P_{m}^{*}(0)}\right)-\right. \\
& \left.\quad-\alpha_{m}\left(\alpha_{m}-\alpha_{m-1}+c_{2 m+1}+c_{2 m+2}\right)\right], \quad m \geqslant 2 .
\end{aligned}
$$

\section{2. $\mu_{1}$ IS SUPPORTED AT A FINITE SUBSET OF THE REAL LINE SYMMETRIC WITH RESPECT TO THE ORIGIN}

For a sake of simplicity, we will consider the set $\{0\} \cup\{ \pm c\}$ as support of the measure $\mu_{1}$. The results obtained in this section can be extended in a natural way to $\{0\} \cup\left\{ \pm c_{k}\right\}_{k=1}^{N}$.

Consider the inner product

$$
\langle p, q\rangle_{s}=\int_{\mathbb{R}} p q \mathrm{~d} \mu_{0}+\lambda_{1} p^{\prime}(0) q^{\prime}(0)+\lambda_{2}\left[p^{\prime}(c) q^{\prime}(c)+p^{\prime}(-c) q^{\prime}(-c)\right], \text { (54) }
$$


where $\mu_{0}$ is a nondiscrete measure supported in an interval of the real line symmetric with respect to the origin so that the corresponding moments of odd order vanish.

Let $\left\{Q_{n}\right\}$ be the sequence of monic polynomials orthogonal with respect to (54). Let $\left\{P_{n}\right\}$ and $\left\{R_{n}\right\}$ be the sequences satisfying (3).

\subsubsection{Orthogonality Measures Associated with $\left\{P_{n}\right\}$ and $\left\{R_{n}\right\}$}

For $n \neq m$,

$$
0=\left\langle Q_{2 n}, Q_{2 m}\right\rangle_{s}=2 \int_{0}^{\infty} P_{n}(x) P_{m}(x) \mathrm{d} \mu_{0}\left(x^{1 / 2}\right)+8 c^{2} \lambda_{2} P_{n}^{\prime}\left(c^{2}\right) P_{m}^{\prime}\left(c^{2}\right) .
$$

Then $\left\{P_{n}\right\}$ is the sequence of monic polynomials orthogonal with respect to the inner product

$$
\langle p, q\rangle_{1}=\int_{0}^{\infty} p q \mathrm{~d} \hat{\mu}_{0}+8 c^{2} \lambda_{2} p^{\prime}\left(c^{2}\right) q^{\prime}\left(c^{2}\right),
$$

where $\mathrm{d} \hat{\mu}_{0}=2 \mathrm{~d} \mu_{0}\left(x^{1 / 2}\right)$.

In the same way, for $n \neq m$

$$
\begin{aligned}
0= & \left\langle Q_{2 n+1}, Q_{2 m+1}\right\rangle_{s} \\
= & \int_{0}^{\infty} x R_{n}(x) R_{m}(x) \mathrm{d} \hat{\mu}_{0}+\lambda_{1} R_{n}(0) R_{m}(0)+2 \lambda_{2} R_{n}\left(c^{2}\right) R_{m}\left(c^{2}\right)+ \\
& \left.+4 \lambda_{2} c^{2}\left[R_{n}^{\prime}\left(c^{2}\right) R_{m}\left(c^{2}\right)+R_{n}\left(c^{2}\right) R_{m}^{\prime}\left(c^{2}\right)\right]+8 \lambda_{2} c^{4} R_{n}^{\prime}\left(c^{2}\right) R_{m}^{\prime}\left(c^{2}\right)\right] .
\end{aligned}
$$

Then $\left\{R_{n}\right\}$ is the sequence of monic polynomials orthogonal with respect to the Sobolev inner product

$$
\begin{aligned}
\langle p, q\rangle_{2}= & \int_{0}^{\infty} x p(x) q(x) \mathrm{d} \hat{\mu}_{0}(x)+\lambda_{1} p(0) q(0)+2 \lambda_{2} p\left(c^{2}\right) q\left(c^{2}\right)+ \\
& +4 \lambda_{2} c^{2}\left[p^{\prime}\left(c^{2}\right) q\left(c^{2}\right)+p\left(c^{2}\right) q^{\prime}\left(c^{2}\right)\right]+8 \lambda_{2} c^{4} p^{\prime}\left(c^{2}\right) q^{\prime}\left(c^{2}\right) .
\end{aligned}
$$

The inner product in (56) can be written in an alternative way as

$$
\begin{aligned}
& \langle p, q\rangle_{2}=\int_{0}^{\infty} x p(x) q(x) \mathrm{d} \hat{\mu}_{0}(x)+\lambda_{1} p(0) q(0)+ \\
& +2 \lambda_{2}\left[p\left(c^{2}\right) \quad p^{\prime}\left(c^{2}\right)\right]\left[\begin{array}{cc}
1 & 2 c^{2} \\
2 c^{2} & 4 c^{4}
\end{array}\right]\left[\begin{array}{c}
q\left(c^{2}\right) \\
q^{\prime}\left(c^{2}\right)
\end{array}\right] .
\end{aligned}
$$

\subsubsection{Algebraic Relations between $\left\{P_{n}\right\}$ and $\left\{R_{n}\right\}$}

For $j<2 n-4$, we get 


$$
\begin{aligned}
& \left\langle\left(x^{5}-\frac{5}{3} c^{2} x^{3}\right) Q_{2 n+1}(x), Q_{j}(x)\right\rangle_{s} \\
& =\left\langle Q_{2 n+1}(x),\left(x^{5}-\frac{5}{3} c^{2} x^{3}\right) Q_{j}(x)\right\rangle_{s}=0,
\end{aligned}
$$

and, as a consequence,

$$
\left(x^{5}-\frac{5}{3} c^{2} x^{3}\right) Q_{2 n+1}(x)=Q_{2 n+6}(x)+\sum_{j=n-2}^{n+2} \alpha_{2 n+1,2 j} Q_{2 j}(x), \quad m \geqslant 1 .
$$

Taking into account (3), from (58) we deduce an algebraic relation between the polynomials $\left\{P_{n}\right\}$ and $\left\{R_{n}\right\}$

$$
\left(x^{3}-\frac{5}{3} c^{2} x^{2}\right) R_{m}(x)=P_{m+3}(x)+\sum_{j=m-2}^{m+2} \alpha_{2 m+1,2 j} P_{j}(x), \quad m \geqslant 1 .
$$

\subsubsection{Recurrence Relations}

Next we prove a result that will be useful to deduce the recurrence relations that the sequences $\left\{P_{n}\right\}$ and $\left\{R_{n}\right\}$ satisfy.

PROPOSITION 9. The multiplication by $x^{4}-2 c^{2} x^{2}$ is a symmetric operator with respect to the inner product (54). Furthermore, it is the polynomial of minimum degree that satisfies such a property.

Proof. The multiplication by a polynomial $h(x)$ is a symmetric operator with respect to the inner product (54) if

$$
\langle h p, q\rangle_{s}=\langle p, h q\rangle_{s} .
$$

Namely,

$$
\begin{aligned}
\lambda_{1}\left[h^{\prime}(0) p(0)+h(0) p^{\prime}(0)\right] q^{\prime}(0)+\lambda_{2}\left[h^{\prime}(c) p(c)+h(c) p^{\prime}(c)\right] q^{\prime}(c)+ \\
\quad+\lambda_{2}\left[h^{\prime}(-c) p(-c)+h(-c) p^{\prime}(-c)\right] q^{\prime}(-c) \\
=\lambda_{1} p^{\prime}(0)\left[h^{\prime}(0) q(0)+h(0) q^{\prime}(0)\right]+\lambda_{2} p^{\prime}(c)\left[h^{\prime}(c) q(c)+h(c) q^{\prime}(c)\right]+ \\
\quad+\lambda_{2} p^{\prime}(-c)\left[h^{\prime}(-c) q(-c)+h(-c) q^{\prime}(-c)\right],
\end{aligned}
$$

for any polynomials $p, q$. This means that

$$
\begin{aligned}
& \lambda_{1} h^{\prime}(0)\left[p(0) q^{\prime}(0)-p^{\prime}(0) q(0)\right]+\lambda_{2} h^{\prime}(c)\left[p(c) q^{\prime}(c)-p^{\prime}(c) q(c)\right]+ \\
& \quad+\lambda_{2} h^{\prime}(-c)\left[p(-c) q^{\prime}(-c)-p^{\prime}(-c) q(-c)\right]=0,
\end{aligned}
$$

for any polynomials $p$ and $q$. When $p(x)=1$, (60) becomes

$$
\lambda_{1} h^{\prime}(0) q^{\prime}(0)+\lambda_{2} h^{\prime}(c) q^{\prime}(c)+\lambda_{2} h^{\prime}(-c) q^{\prime}(-c)=0 .
$$

Taking $q(x)=x, x^{2}, x^{3}$, respectively, we get 
1. $\lambda_{1} h^{\prime}(0)+\lambda_{2} h^{\prime}(c)+\lambda_{2} h^{\prime}(-c)=0$,

2. $2 c \lambda_{2}\left[h^{\prime}(c)-h^{\prime}(-c)\right]=0$,

3. $3 c^{2} \lambda_{2}\left[h^{\prime}(c)+h^{\prime}(-c)\right]=0$.

Hence

$$
h^{\prime}(c)=h^{\prime}(-c)=h^{\prime}(0)=0 .
$$

This means that the polynomial $h(x)$ of minimal degree satisfies

$$
h^{\prime}(x)=x(x-c)(x+c),
$$

and, as a consequence,

$$
h(x)=\frac{x^{4}}{4}-\frac{c^{2} x^{2}}{2} .
$$

If $h$ is chosen to be monic, then

$$
h(x)=x^{4}-2 c^{2} x^{2} .
$$

PROPOSITION 10. Consider a symmetrized Sobolev inner product as in (54). Let $\left\{Q_{n}\right\}$ be the corresponding sequence of monic orthogonal polynomials. Assume that $\left\{S_{n}\right\}$ and $\left\{R_{n}\right\}$ are the sequences such that (6) holds. Then, the following recurrence relations are obtained.

$$
\begin{aligned}
& \left(x^{4}-2 c^{2} x^{2}\right) Q_{n}(x)=Q_{n+4}(x)+\sum_{j=n-4}^{n+3} \alpha_{n j} Q_{j}(x), \quad n \geqslant 4 \\
& \left(x^{2}-2 c^{2} x\right) P_{n}(x)=P_{n+2}(x)+\sum_{j=0}^{3} \alpha_{2 n, 2(n-2+j)} P_{n-2+j}(x), \quad n \geqslant 2 \\
& \left(x^{2}-2 c^{2} x\right) R_{n}(x)=R_{n+2}(x)+\sum_{j=0}^{3} \alpha_{2 n+1,2(n-2+j)+1} R_{n-2+j}(x), \quad n \geqslant 2 .
\end{aligned}
$$

Proof. From Proposition 9, for $0 \leqslant j<n-4$, we get

$$
\left\langle\left(x^{4}-2 c^{2} x^{2}\right) Q_{n}, Q_{j}\right\rangle_{s}=\left\langle Q_{n},\left(x^{4}-2 c^{2} x^{2}\right) Q_{j}\right\rangle_{s}=0,
$$

which yields (61).

For $n=2 m$, (61) becomes (62). For $n=2 m+1$ (61) becomes (63).

Therefore, the symmetric components $\left\{P_{n}\right\}$ and $\left\{R_{n}\right\}$ are Sobolev polynomials and they satisfy the five-term recurrence relations given in (62) and (63).

\section{Acknowledgements}

The work of the second author has been partially supported by KRF-2002-070C00004. The work of the third author has been partially supported by Dirección 
General de Investigación (Ministerio de Ciencia y Tecnología) of Spain under grant BFM 2000-0206-C04-01 and INTAS project INTAS 2000-272.

This paper was finished during the second author's visit to Universidad Carlos III under the Sabbatical Program supported by Vicerrectorado de Investigación of this University.

The authors thank the referees for their valuable comments, suggestions, and remarks in order to improve the presentation and the contents of the manuscript.

\section{References}

Alfaro, M., Marcellán, F., Meijer, H. G. and Rezola, M. L. (1994) Symmetric orthogonal polynomials for Sobolev-type inner products, J. Math. Anal. Appl. 184, 360-381.

Alfaro, M., Pérez, T. E., Piñar, M. A. and Rezola, M. L. (1999) Sobolev orthogonal polynomials: The discrete-continuous case, Methods Appl. Anal. 6, 593-616.

Arvesú, J., Atia, J. and Marcellán, F. (2002) On semiclassical linear functionals: The symmetric companion, Comm. Anal. Theory Contin. Fractions 10, 13-29.

Bavinck, H. and Meijer, H. G. (1989) Orthogonal polynomials with respect to a symmetric inner product involving derivatives, Appl. Anal. 33, 103-117.

Bavinck, H. and Meijer, H. G. (1990) On orthogonal polynomials with respect to an inner product involving derivatives: Zeros and recurrence relations, Indag. Math. (N S) 1, 7-14.

Bueno, M. I. and Marcellán, F. (2003) Continuous symmetric Sobolev inner products, Internat. Math. J. 3, 319-342.

Cachafeiro, A., Marcellán, F. and Moreno-Balcázar, J. J. (2003) On asymptotic properties of FreudSobolev orthogonal polynomials, J. Approx. Theory 125, 26-41.

Chihara, T. S. (1978) An Introduction to Orthogonal Polynomials, Gordon and Breach, New York.

Evans, W. D., Littlejohn, L. L., Marcellán, F., Markett, C. and Ronveaux, A. (1995) On recurrence relations for Sobolev orthogonal polynomials, SIAM J. Math. Anal. 26, 446-467.

Gautschi, W. (1982) An algorithmic implementation of the generalized Christoffel theorem, In: G. Hämmerlin (ed.), Numerical Integration, Internat. Ser. Numer. Math. 57, Birkhäuser, Basel, pp. 89-106.

Hendriksen, E. and van Rossum, H. (1985) Semiclassical orthogonal polynomials, In: C. Brezinski et al. (eds), Polynômes Orthogonaux et Applications, Lecture Notes in Math. 1171, Springer-Verlag, Berlin, pp. 354-361.

Jung, I. H., Kwon, K. H. and Lee, J. K. (1997) Sobolev orthogonal polynomials relative to $\lambda p(c) q(c)+\left\langle\tau, p^{\prime}(x) q^{\prime}(x)\right\rangle$, Comm. Korean Math. Soc. 12, 603-617.

Kim, D. H., Kwon, K. H., Marcellán, F. and Yoon, G. J. (2002) Sobolev orthogonality and coherent pairs of moment functionals: An inverse problem, Internat. Math. J. 2, 877-888.

Maroni P. (1991) Une théorie algébrique des polynómes orthogonaux. Application aux polynômes orthogonaux semiclassiques, In: C. Brezinski et al. (eds), Orthogonal Polynomials and Their Applications, IMACS Annals on Comp. and Appl. Math. 9, pp. 95-130.

Meijer, H. G. (1997) Determination of all coherent pairs of measures, J. Approx. Theory 89, 321-343.

Nevai, P. (1983) Orthogonal polynomials associated with $\exp \left(-x^{4}\right)$, Proc. Canad. Math. Soc. 3, 263-285.

Nevai, P. (1984) Asymptotics for orthogonal polynomials associated with $\exp \left(-x^{4}\right)$, SIAM J. Math. Anal. 15, 1177-1187.

Pérez, T. E. and Piñar, M. A. (1996) On Sobolev orthogonality for generalized laguerre polynomials, J. Approx. Theory 86, 278-285. 